\title{
DESIGUALDADES DE GÉNERO Y ESTRATEGIAS DE PARIDAD EN LA EDUCACIÓN: RESULTADOS DE UNA INVESTIGACIÓN
}

\section{Gender Inequalities and Equality Strategies in Education: Results of a Research}

\author{
Manuel Carlos Silva \\ mcsilva2008@gmail.com \\ Universidade do Minho - Portugal
}

Recibido: 28-02-2017

Aceptado: 20-04-2016

\section{Resumen}

Varios estudios apuntan hacia la reproducción de la desigualdad por territorio, clase y género, destacando la discriminación femenina en el pasado, especialmente en Portugal, con altas tasas de analfabetismo, sobre todo durante el Estado Novo. Las prioridades de la política educativa desde 1974 han producido una reducción de las disparidades educativas. En este texto, se exponen algunos resultados de un proyecto de investigación, en el que se comprueba la hipótesis de trabajo de que cuanto mejor provistas de recursos están las famílias más cualificados son los individuos y cuanto menos equipadas y con más edad menos cualificados son, así como cuanto más jovenes más cualificados son. Más, se verifica entre las mujeres una más intensa movilidad educativa, aunque no profesional, que entre los hombres.

Palabras Clave: Género, desigualdades, educación, profesión, edad.

\begin{abstract}
Several studies sustain the thesis of reproduction of inequalites by territory, class and gender, highlighting female discrimination in the past, namely in Portugal, with high rates of illiteracy, specially during the Estado Novo of Salazar dictatorship. The priorities of education policies since 1974 have produced a reduction of educational disparities. In this text, I will give account of some results of a research project, in which is verified that the working hypothesis that the more wellresourced are the families more qualified the individuals are and the less well-resourced and older less qualified they are, as well as younger people are more qualified. In addition, among women, there is a more intense educational mobility, although not a profesional one, that among men.
\end{abstract}

Keywords: Gender, inequalities, education, profession, age. 


\section{Introducción}

Las desigualdades de género en la educación han sido desde hace algunas décadas objeto de estudio, constituyento todavía un campo relativamente reciente. Varios estudios han evidenciado la existencia de la desigualdad de género en términos de acceso y éxito educacional, señalando especialmente a la discriminación femenina en los itinerarios educacionales en el pasado. Esta desigualdad ocurría en Portugal, un país donde la implementación de la educación básica universal pasó por difíciles fases de consolidación. Aunque las declaraciones alrededor de la educación obligatoria daten de la primera mitad del siglo X1X, el documento legal sobre la misma no apareció hasta final de ese siglo (Araújo, 2000). Las elevadas tasas de analfabetismo se mantuvieron durante el siglo XX, en particular durante el Estado Novo (en 1971 se situaban aún en el 75\%). Esta situación ha condicionado las tendencias de escolarización de las generaciones posteriores, colocando Portugal en la cola de Europa (Sebastião, 1998).

En el ámbito de las desigualdades de género en la educación, las cuestiones más relevantes se pueden enunciar del siguiente modo: ¿Cómo entender y explicar no sólo el relativo retraso en la escolarización en comparación con otros países como el recurrente fenómeno histórico de la relativa descalificación de las mujeres en relación a los hombres?¿Hasta qué punto se verifica una reversión de la situación en el sentido de la paridad educativa y, en caso afirmativo, desde cuándo y cuáles son sus contornos? ¿Cuáles son las razones y los efectos e implicaciones de esta evolución?

En primer lugar, cabe señalar que desde el 25 de abril de 1974 se han registrado un conjunto de transformaciones importantes, debiendo observarse que la escolarización de la población ha sido una de las prioridades de las políticas educativas en las últimas décadas en un intento de acercarse a los estándares de la Comunidad Europea. Aunque se constate la inversión en la elevación de los niveles de escolarización, este proceso se ha caracterizado por el no cumplimiento de todas las metas formuladas (A.N. Almeida y Vieira, 2006). No obstante, Portugal consigue en tres décadas y de forma acelerada elevar la escolarización de las personas, sobre todo en el primer y segundo ciclo de educación básica y, más recientemente, en el tercer ciclo y en la enseñanza secundaria y superior.

Como se ha señalado ya, en un pasado reciente eran las mujeres las más afectadas por la débil democratización de la enseñanza superior, constatándose que

"[...] muchas chicas con estudios más allá de la escolaridad obligatoria se quedaban al final de la enseñanza secundaria, frecuentaban poco la formación profesional y las pocas que lo hacían se deparaban con una oferta formativa mayoritariamente dirigida a las profesiones tradicionalmente masculinas, intentando o no acceder a un empleo, lo cierto es que la probabilidad de lo conseguieren era, por esas razones, bastante reducida" (Chagas y Perista, 2010:194). 
Desde fines de los años 1980 se han promovido esfuerzos para reducir las disparidades entre hombres y mujeres en la educación y en el empleo. En la década de 1990, hay una presencia más significativa de las mujeres en la enseñanza superior, a pesar de que se continúan constatando campos de estudio, en que las mujeres están menos representadas, como, por ejemplo, en Arquitectura (31\%) y en Ingeniería (44\%), en el año académico de 1996-97 (Chagas y Perista, 2010). Datos relativos a 2004 apuntaban ya hacia una tasa de feminización superior en la educación superior (ibidem). Con respecto al aprovechamiento, y según datos referentes a 2007/2008, se observan tasas más altas de mujeres graduadas en educación superior o de maestría o incluso de doctorado (Araújo, 2010).

El panorama general en el resto de Europa es el de que las mujeres tienen niveles de escolarización superiores a los de los hombres (Eurostat, 2005). La variable edad interfiere bastante en la comprensión de estos datos, una vez que, si nos fijamos en las mujeres de un nivel mayor de edad, podemos verificar precisamente, en general, lo contrario, o sea, un menor nivel de escolarización. El fenómeno se relaciona simultáneamente con la creciente femenización de las universidades europeas y la importancia de un título universitario en la reducción de las desigualdades de género en el acceso al empleo. La inversión en la carrera profesional se revela preponderante para las mujeres más jóvenes, siendo el nivel educativo explicativo de una mayor participación de las mujeres en el trabajo.

\section{Igualdad de oportunidades: entre la reproducción y movilidad educacional}

Uno de los tópicos nucleares de esta investigación se ha centrado en la cuestión de la igualdad de oportunidades que, en el caso en cuestión, incide sobre la igualdad de oportunidades por género. En primer lugar, conviene resituar la problemática a dos niveles: un primero sobre el concepto de igualdad de oportunidades, sus raíces teóricas e ideológicas, lo que no podrá ser llevado a cabo sin implicar, en contrapunto, la desigualdad de oportunidades, lo que plantea la pertinente cuestión: ¿en qué medida es posible separar la cuestión de la igualdad de oportunidades por género de la cuestión preliminar de la (des)igualdad de oportunidades tout court? Sin menospreciar la especificidad del problema de género, como veremos, el problema de la (des)igualdad de oportunidades convoca una serie de teorías que no es posible descartar y que están ligadas a la temática de las precondiciones o prerequisitos básicos acerca de la igualdad de oportunidades, cuya ausencia o presencia y grado de la misma remite a la problematización de los procesos de manutención/reproducción de las desigualdades o su superación/reducción, ora aún para la transformación social de la estructura, ora por la movilidad social. Pero ¿de quién? ésta es la cuestión central. Buscando especificar: ¿en que medida el actual sistema económico, social y político en efecto reproduce las desigualdades preexistentes o permite el cambio de estas situaciones? Y, si lo hace, ¿cuál es su significado y alcance? ¿Se trata de una transformación de 
fondo que abre las avenidas de la movilidad social a todos los ciudadanos y ciudadanas en el goce de sus derechos y libertades fundamentales o se limita a un número más o menos restringido de grupos e individuos?

La cuestión central subyacente a la problemática de la reproducción y/o movilidad social es cómo se distribuyen los recursos y las recompensas entre los ciudadanos de diferentes clases, etnias y géneros, cómo los portadores de estas condiciones se reproducen intra e intergeracionalmente en su propio seno y/o se conocen procesos de movilidad vertical sea en sentido descendiente sea en sentido ascendiente.

Sobre esta cuestión han sido perfiladas varias respuestas teóricas, que, de forma sintética, importa enunciar y exponer. Así, autores como Parsons (1988), Dahrendorf (1959), Bell (1975), considerando las sociedades modernas (suficientemente) democráticas y abiertas hasta el punto de que, a través de la educación y por mérito del individuo, ofrecieran igualdad de oportunidades y seleccionaran los mejores, sostienen que la movilidad social de los individuos es el rasgo dominante de esas sociedades. En otras palabras, contrariamente a las sociedades tradicionales, en las que el estatuto de cada individuo se configura en la base de las relaciones de sangre, familiares o de compadrazgo, en las sociedades modernas la movilidad es el hecho recorriente, lo que implica, en gran parte, la negación del principio de la reproducción social basada en criterios de clase o adscriptivos.

En esta óptica, si, por un lado, las sociedades modernas conocen una mayor nivelación social que en las anteriores sociedades, por el otro, esto no implica que se camine en la dirección de la igualdad social, que, según estos y otros autores, es utópico e imposible. Además, la posición teórica de la inevitabilidad de las desigualdades sociales, dado como algo inherente y universal para todas las sociedades, conociendo varias justificaciones a lo largo del tiempo, llegó a ser trabajada por la corriente (estructural)funcionalista, principalmente por Davis y Moore (1976) y, de modo más sistémico, por Parsons (1988) y Merton (1970). Desde esta perspectiva, las desigualdades sociales se justifican desde la asunción de la existencia de un vínculo directo entre el mérito individual y las recompensas ofrecidas por la sociedad en contraste con los criterios adscriptivos de nacimiento, parentales o clientelares, legitimadores de las desigualdades del viejo orden tradicional. Más, según Dahrendorf $(1959,1990)$, entre otros, los procesos posicionales y procesos de movilidad resultarían de las capacidades y acciones diferenciadas de los individuos en el aprovechamiento o no de las oportunidades ofrecidas.

Hay en estos autores una acentuación del papel dinámico de los actores sociales, en cuanto configuradores de las propias estructuras sociales, alegadamente en la estela de Weber (1978). Por otra parte, esta posición no es defendida ni exclusiva ni principalmente por los estructurofuncionalistas - que asumen que las normas y los valores del sistema social son los principios constituyentes de los diversos status y roles - sino también por las corrientes accionalistas, particularmente por neoweberianos como Dahrendorf (1993) y Parkin (1979) que ponen de relieve las relaciones de poder como un factor decisivo de los diferentes lugares, así como por la actual perspectiva interaccionista, aunque ya alejada de las premisas estructuro-funcionales y 
subrayando más los procesos de interacción, negociación y concertación entre los actores sociales (cfr. Goffman, 1974).

En un polo opuesto se sitúan las teorías marxistas o marxisantes (Althusser, 1972; Poulantzas, 1975; Bertaux, 1977) y, en una primera fase, Bourdieu y Passeron (1970) que sostienen, unos de modo (casi)absoluto y categórico, otros sólo como regla tendencial que las desigualdades sociales, fundamentalmente las condiciones de clase y de género, se reproducen intra e intergeracionalmente. Eventuales cambios horizontales de sector a sector no confirmarían esta tesis, cuyos defensores parten de la premisa de que, no alterándose las premisas básicas de las desigualdades sociales inherentes a ciertos sistemas, las diversas instituciones y mecanismos jurídicos, socio-económicos y políticos (casa, propiedad, herencia, migraciones y sistema educativo y judicial, partidos) tenderán a reproducir las desigualdades sociales. Sin embargo, desde este punto de vista, si, por un lado, se afirma el principio de la reproducción social intra e intergeneracional, por otro no asume la desigualdad social como una fatalidad de la naturaleza humana ni siquiera como efecto del principio meritocrático sino más bien como resultado de un determinado grado de acceso y posesión de determinados recursos principalmente de los medios de producción en diversos modos de producción y de la correspondiente dominación de clase por parte de las clases explotadoras sobre las dominadas y explotadas a lo largo de la historia. Según Poulantzas (1975), el aspecto fundamental de la reproducción de las relaciones sociales no es el de los 'agentes', sino de la reproducción de las clases sociales. Es destacada, en esta interpretación marxista, la amplia prevalencia de la estructura sobre la agencia - los actores sociales.

El debate centrado entre la primera y segunda posición ha sido algo sesgado e incluso contaminado por la interferencia de justificaciones ideológicas. Mientras a la posición de los teóricos de la reproducción social subyace la idea de que las desigualdades sociales, en particular de clase, constituyendo una consecuencia del funcionamento de modos de producción explotadores y opresivos, sólo pueden ser eliminadas a través de la socialización de los medios de producción y el establecimiento de una sociedad socialista, los defensores de la tesis de la movilidad social (intra/inter)generacional se basan en la suposición de que cada individuo es remunerado en su función según su talento o mérito y, por lo tanto, la sociedad moderna actual, sin eliminar las desigualdades sociales, permite a todos igualdad de oportunidades y, por tanto, posibilidad legal y real de ascensión social. En el fondo, esta posición pretende, de modo subliminal y subrepticio, vehicular la ideología de que, si los indivíduos quisieran e invirtieran en ese sentido, podrán, al acceder a los criterios existentes y al conformarse con las pautas dominantes y las normas vigentes, ascender socialmente. Es por esta razón que los marxistas rechazan la teoría de la movilidad social como una regla, acusando a sus defensores de constituir una forma de alienación político-ideológica favorable a la burguesía, en la medida en que crea expectativas irrealistas e irrealizables para la aplastante mayoría de los miembros de las clases asalariadas o desprovistas de recursos. Por eso, aún incluso cuando esto puede constituir una parte integrante de la ideología burguesa, se trata de deslindar lo real de lo ideológico y contrastar hasta 
qué punto se verifica o no un cierto grado de movilidad, sin caer en otro punto de partida, también él apriorístico e ideológico.

Otros autores, neo-marxistas o críticos del marxismo tradicional (Bourdieu, 1980; Wright, 1985; Bader y Benschop, 1988; Benschop, 1993; Silva 1998, 2009), como otros post-marxistas y/o neoweberianos como Giddens (1981), Goldthorpe (1987) y Estanque y Mendes (1997), aceptando grosso modo la tesis de la reproducción social, admiten todavía, principalmente en las sociedades modernas o en transición hacia la modernidad, un mayor o menor grado de movilidad social no sólo en el sentido descendente - lo que es además reafirmado por marxistas en relación a procesos de proletarización e incluso, más o menos visibles, al menos en el largo plazo, en diversos contextos históricos, sino también hacia arriba, lo que, aunque limitado, también ocurre $y$, en el caso en cuestión, en relación a estrategias de paridad e igualdad de género. Aplicando esta tesis a la cuestión de género, esto implicaría que determinada estructura social permite la reproducción y/o el refuerzo social de la mayoría de los miembros de cada grupo/categoría social en su medio pero admite que, bajo ciertas circunstancias proporcionadas por factores institucionales, por movilidad espacial y sus oportunidades de vida, empoderamiento e inversión de ciertas capacidades, habilidades y otros recursos, que pueda influir alterando las trayectorias biográficas hasta el punto de convertirse en un ascenso social. Del mismo modo, la concurrencia de ciertos ambientes adversos, falta de oportunidades y de ciertos reveses (epidemia, enfermedad, paro, divorcio, persecución política) pueden resultar en una caída social. En esta perspectiva, se trata de, sin perder de vista los constreñimientos estructurales y la lógica de la reproducción social, relevar las trayectorias sociales de los actores sociales - sea, por ejemplo, el de la mujer emprendedora en situación de relativa autonomía, sea, en la mayoría de los casos, como asalariada en las posiciones de desventaja, exceptuando sin embargo casos minoritarios que, en una orientación estratégica en base familiar, hayan creado condiciones para moverse socialmente y/o contribuído a la transformación social.

En principio y teniendo en cuenta datos empíricos obtenidos en esta investigación, asumo esta tercera posición, que indica una perspectiva teórica crítica de prácticas meritocráticas. O sea, sosteniendo el marco teórico de la tesis de la reproducción social, la cual se aplica a la mayoría de las familias e individuos, los datos recogidos ayudan a confirmar que, a la par de situaciones de movilidad descendente, se verifican situaciones y casos que, en mayor o menor grado, han conocido mejoras sociales e incluso algunos casos de movilidad ascendente. Es decir, en la medida en que perfilamos una mirada teórica de orientación neo-marxista cruzada con las ricas contribuciones weberiana e interaccionista, nos encontramos en una posición de apertura a la combinación de elementos teóricos que permiten precisamente medir y evaluar las situaciones de reproducción, los tipos y grados de movilidad social. Éstos dependen y varían de sociedad a sociedad y sin duda, con el tiempo, de lugar a lugar y periodo histórico y, en particular, por la combinación de factores políticos e institucionales a nivel nacional y sobre todo europeo, especialmente, con sus programas en favor de la paridad de género. 
Con la revisitación del debate en torno a la reproducción versus movilidad social podría parecer algo inoportuno empezar por ecuacionar el problema en estos términos y, en un primer momento, no luego a partir de las desigualdades de género, así como de su ligazón con el principio de igualdad de oportunidades de género, sino justamente por la cuestión más amplia, completa y profunda de las desigualdades sociales, sus diversos tipos y, en particular, las de classe. En este sentido, la mujer en cuanto actora social, trabajadora y madre, frecuentemente cuidadora en la casa, es doblemente víctima de la desigualdad social en los procesos de reproducción social (cf. Harris y Young, 1981). Como veremos, a propósito del análisis de los datos empíricos, este punto de partida irá ciertamente a proporcionar una vía de comprensión, interpretación y explicación de las situaciones de explotación, jerarquización y dominación, que no pasan tan sólo por la desigualdad de género, sino que con ella coexisten e incluso a veces se superponen otras formas de desigualdad como las de clase, étnicas u otras fundamentalmente en términos de percepción social y conciencia por parte de las mujeres, particularmente en el contexto laboral.

Sólo articulando la problematización de las desigualdades sociales, en general, con la específica desigualdad de género, se podrá enmarcar, entender el mayor o menor énfasis dado no solo por los hombres como sobre todo por las propias mujeres a las diversas formas de trato desigual por género. Tal como fue posible concluir en otro estudio realizado por Marques, Silva y Veiga (2006), son precisamente las desigualdades de clase las que tienden a diluir, en algunos contextos, las de género, no porque éstas sean menos importantes social y políticamente, sino porque resaltan relativamente menos que las de clase. Concretando, los salarios de hombres y mujeres en Barcelos, en un contexto de industrialización difusa, son tan bajos que este es el hecho social más sobresaliente en la percepcion de las mujeres, a pesar de obtener un salario inferior a los de los hombres en $20 \%$. El salario es percebido como un componente del ingreso familiar. Tales situaciones son propiciadoras de una amortiguación de la conflictualidad y demanda laborales o incluso de consentimento con el estado de cosas, sea desde el prisma de las relaciones de género, sea de las relaciones de explotación y dominación en términos de clase, tal como señala y desarrolla Burawoy (1979) y, en Portugal, Silva $(1998,2009)$ en el contexto rural de la región de Minho sobre el campesinado e Estanque (1999) en el industrial, designadamente entre los trabajadores del calzado en S. João da Madeira. Pero pasemos ahora al análisis de las características educativas de los participantes en la muestra y sus similitudes o diferencias con las de la población portuguesa.

\section{Entre las desigualdades y la paridad de género en la educación: una evolución positiva}

Una variable relevante de la caracterización de la muestra es la que se refiere a la distribución global de los encuestados por nivel de educación. Según los datos de la encuesta, se señala la presencia, aunque baja, de personas analfabetas (3.4\%). Éstas, agregadas a personas que, aunque 
sabiendo leer y escribir, no han terminado el cuarto grado, constituyen una tasa no despreciable del 10,2\%. Sin embargo, la mayor parte de las personas encuestadas se sitúa en los niveles bajos e intermedios de la escolarización - 1er, $2^{\circ}$ y 3er ciclo - las cuales suman un $66.7 \%$, lo que es indicativo de una población poco cualificada, siendo la moda el ciclo básico.

La enseñanza secundaria es poseída por un $14 \%$ de los encuestados, los cuales, agregados a $\operatorname{los} 1.2 \%$ de los titulares poseedores de curso profesional o medio, completan un $15.2 \%$. Por último, las personas con una educación superior, postgrado y maestrías conforman el $7.8 \%$, contrariando la idea de que estamos ante una masificación de la educación superior. Cruzando la edad, o más precisamente, los grupos de edad por sexo, obtenemos los siguientes resultados:

\section{Gráfico 1. Escolarización por sexo (\%)}

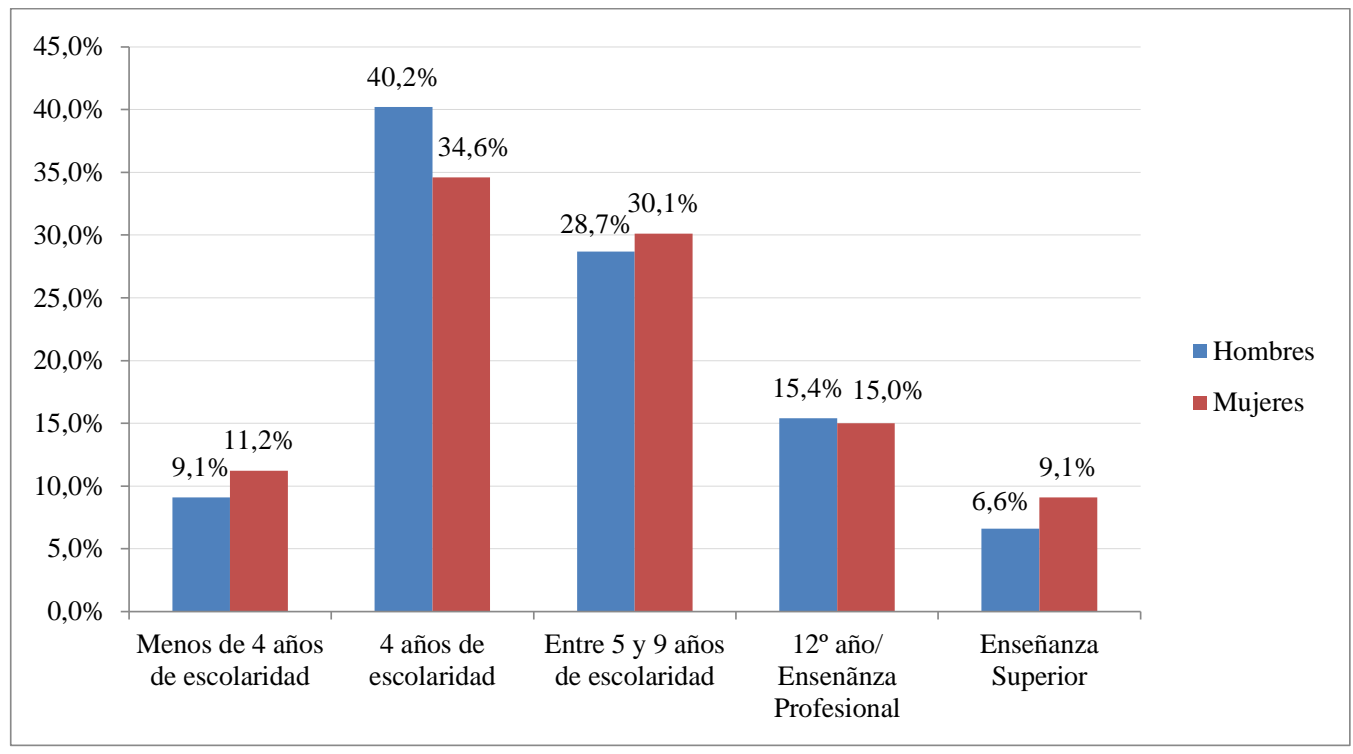

Fuente: IDG, 2011; N=802 (383 Hombres; 419 Mujeres)

Exceptuando la categoría de los indivíduos con 4 años de escolarización en que se verifica una ventaja de los hombres sobre las mujeres (40\% vs 35\%), más frecuente en las categorías de mayor edad, cabe señalar que en las demás o se verifica un empate o un ligero predominio de mujeres en la educación, más presente entre las generaciones más jóvenes. Esta tendencia corrobora la tesis de la femenización de la enseñanza secundaria y superior. Veamos, por ejemplo, cómo los datos relacionados con el año escolar 2007/2008 indican una tasa de femenización del $52,6 \%$ en educación secundaria y un 53,5\% en la educación superior (Araújo, 2010). Estudios centrados en estas cuestiones (cf. Costa y Machado, 2007; Araújo 2010) dan cuenta de que las mujeres ingresan mayoritariamente en las universidades y concluyen sus cursos con mayor éxito.

Si cruzamos los grupos de edad con la educación de los encuestados, obtenemos los siguientes resultados expuestos en el gráfico 2 : 


\section{Gráfico 2. Escolarización por grupos de edad: hombres (\%)}

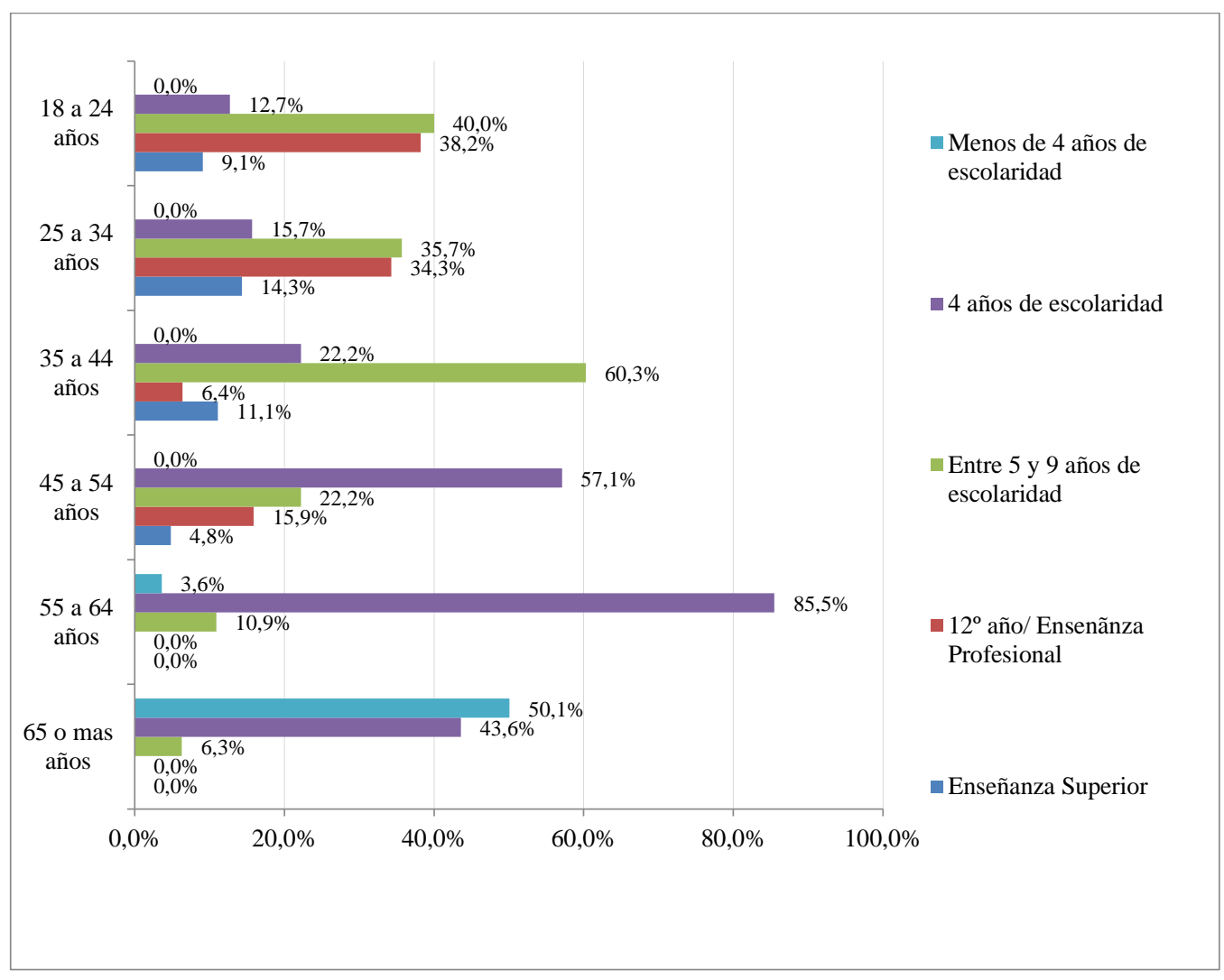

Fuente: IDG, 2011; N=383

Del gráfico 2 se infiere que los detentores del diploma de enseñanza superior se sitúan menos en los grupos de mayor edad $(4,8 \%)$ y más en los grupos intermedios $(11,1 \%)$ y sobre todo en los grupos más jovenes, a saber, de los 18 a los 34 años, con el 23,4\%. La reducida proporción de mayores de edad con formación superior puede ser explicada por condicionantes estructurales, en particular, el cierre socioeconómico que estaba vigente durante el régimen dictatorial portugués en el acceso a la educación. Lo mismo se puede decir y con mayor énfasis sobre quien detenta el $12^{\circ}$ año o curso profesional - el 72,5\% entre los 18 a los 34 años versus el 22,2\% de personas con 35 o más años -, aunque practicamente sin significación más allá de los 55 años. Entre el $5^{\circ}$ y el $9^{\circ}$ año se sitúan personas en los grupos de edad intermedios, aunque hay también una relativa dispersión por otros grupos. En este caso nos encontramos con un segmento poblacional que disfruta de circunstancias de democratización de la enseñanza superior, lo que refleja las tasas más elevadas de escolarización.

Cruzando la escolarización de las mujeres por grupos de edad, obtenemos la siguiente distribución (cf. gráfico 3): 


\section{Gráfico 3. Escolarización por grupos de edad: mujeres (\%)}

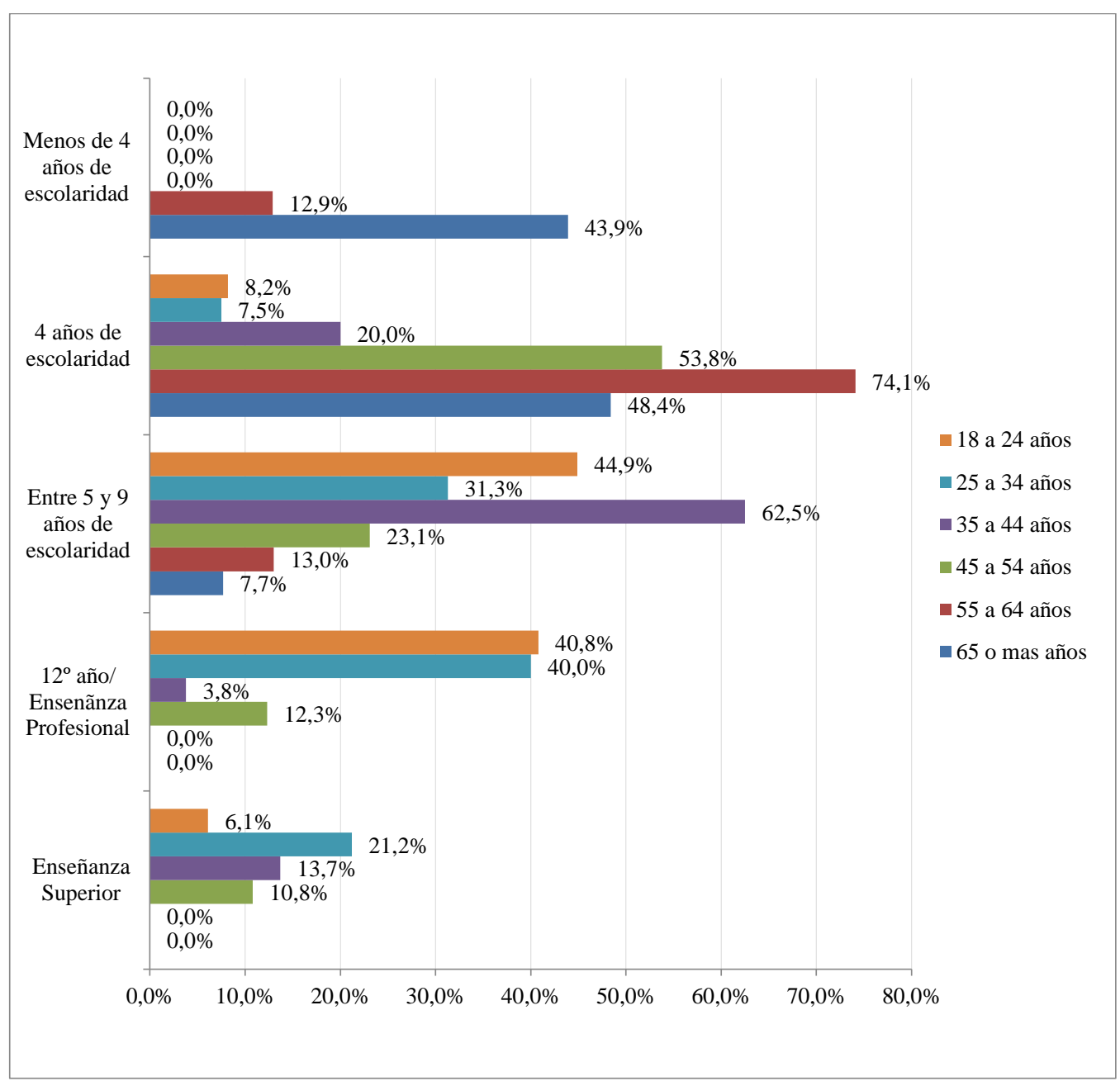

Fuente: IDG, 2011; $N=419$

En el gráfico 3 se puede leer una relación similar a la encontrada en la educación con los grupos de edad masculinos: se constata que las poseedoras del diploma de educación superior se sitúan en los grupos de edad menos elevados, con una fuerte presencia en los niveles intermedios y, sobre todo, en las filas de las más jovenes, es decir, de los 18 a los 34 años. En cualquier caso, con respecto a las mujeres que tienen curso superior o profesional, también hay una fuerte expresión en las filas de las más jóvenes: el 80,8\% entre 18 a 34 años frente a 16,1\% con 35 o más años, de los cuales $12,3 \%$ por encima de 45 y más años. Entre el quinto y noveno grado son personas en las edades intermedias y superiores: entre 35 y 44 años, aunque también entre los jóvenes. Por último, con 4 años de escolarización y sobre todo con menos de 4 años de la misma, se destacan en gran medida las categorías de edad mayores de 55 años y, sobre todo, con 65 o 
más años. Estos datos llevan a la conclusión de que cuanto más se eleva en los niveles de edad, menos cualificados en la educación y a la inversa: los niveles más jovenes se sitúan más alto en las calificaciones y con mayor porcentaje. Esta tendencia contraría la observada en los grupos de edad mayores, donde sobre todo son las mujeres quien tienen menor nível de educación que los hombres. Sin embargo, las mujeres que nacieron después de 1955 muestran ya un trayecto educativo muy diferenciado de las escuelas anteriores, contribuyendo a la situación verificada en 2001: la educación de las mujeres en el grupo de edad de 25 a 34 años es, por comparación com los hombres, superior en la educación secundaria y superior (Resende y Vieira, 2003).

Con respecto a la escolarización de los hombres-padres de los encuestados, analizándola por niveles de educación, constatamos que, exceptuando el $5.1 \%$ que poseen el diploma de enseñanza superior y el $11.1 \%$ que terminó el $12^{\circ}$ año o un curso profesional, otros, en su gran mayoría, tienen baja o muy baja cualificación: el 18,3\% entre 5 y 9 años, el 42,9\% con 4 años de escolarización y el 22,6\% con menos de 4 años. Estos resultados son indicativos de un volumen considerable de bajas calificaciones, comprobándose lo mismo en la figura 8, más específicamente en los niveles de la educación de los padres de los encuestados:

\section{Gráfico 4. Escolarización de los padres de los/as encuestados/as (\%)}

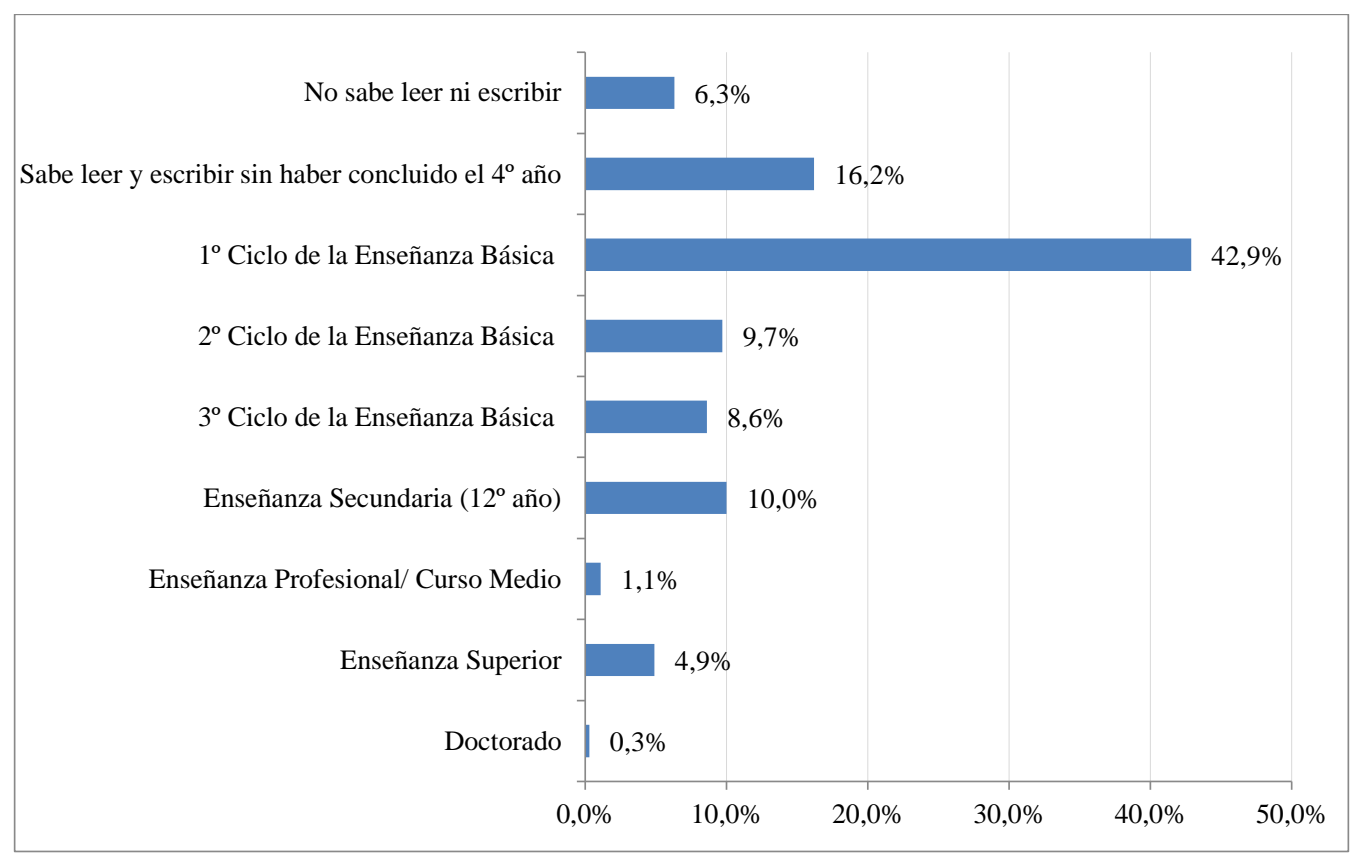

Fuente: IDG, 2011; N=371

Tal como se puede desprender del gráfico 4, a excepción del 0,3\%, del 4,9\% y del 1,1\% que poseen respectivamente doctorado, licenciatura y curso medio/profesional, seguido del $10 \%$ com la enseñanza secundaria, la gran mayoría detenta bajas calificaciones: $18,3 \% \operatorname{com} 6^{\circ} \mathrm{y} / \mathrm{o} 9^{\circ}$ año, 
el 42,9\% con enseñanza básica, el 16,2\% que, aunque sepa leer y escribir, no ha frecuentado la escuela básica y el 6,5\% de analfabetos en sentido literal.

A fin de comparar el nivel de escolarización de las madres de los encuestados/as, el gráfico 5 nos ofrece un mapa de su distribución:

\section{Gráfico 5. Escolarización de las madres de lo/as encuestados/as (\%)}

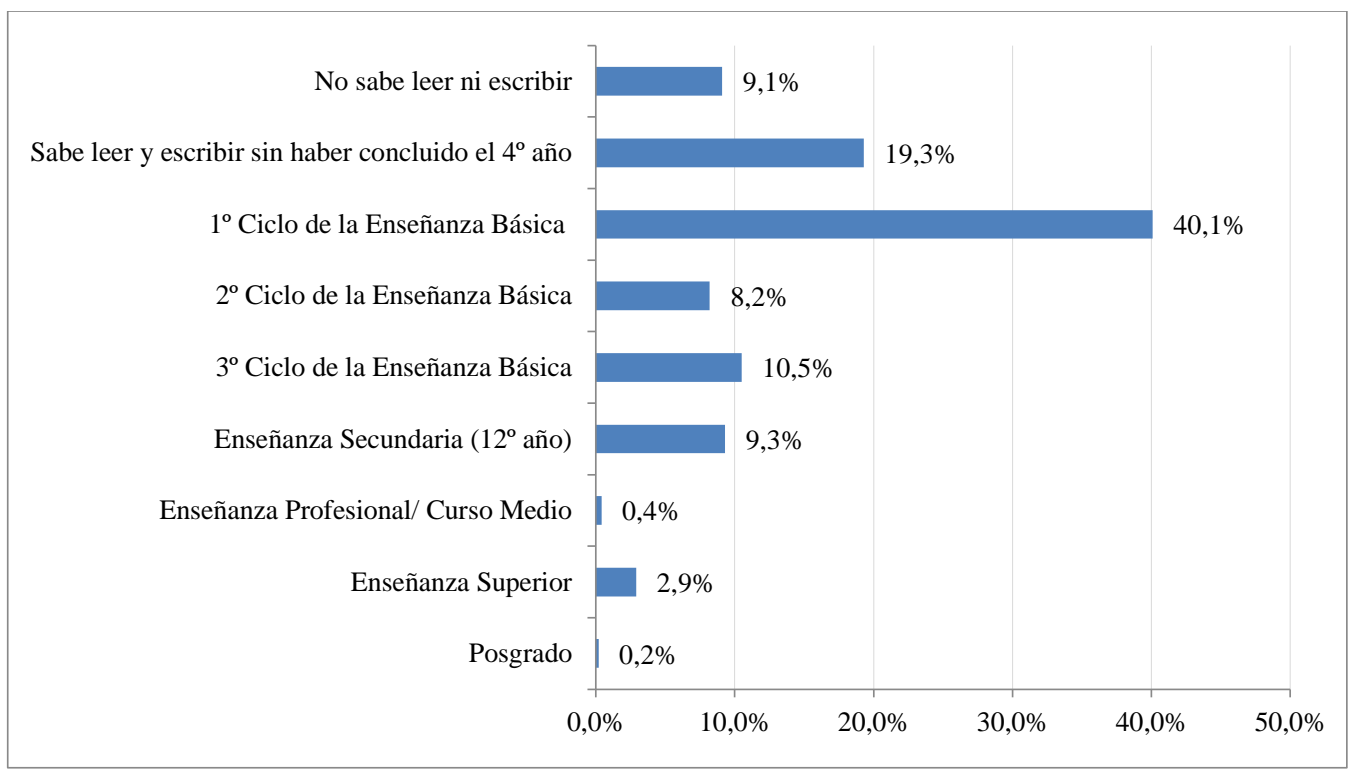

Fuente: IDG, 2011; N=486

Tratándose del caso de las madres de los encuestados/as, los resultados son indicativos de una alta tasa de analfabetismo $(9,1 \%)$ y baja escolarización con el $48 \%$ (40,1\% con cuarto grado y $8.2 \%$ con el 6 año de edad o $2^{\circ}$ año), haciendo un total del $57 \%$ de las madres de los encuestados. Se siguen las franjas intermedias con el $19,8 \%$ contabilizando el grado $9^{\circ}$ y $12^{\circ}$ grado, pero a partir de ahí son verdaderamente residuales, es decir, solo el 0,4\% de las mujeres tenían curso profesional y $3,1 \%$ postgrado. Reagrupando los datos por niveles de enseñanza, se constata que las madres de los encuestados/as están en niveles bajos o muy bajos de escolarización. Dicho de otra manera, contabilizando las que tuvieron menos de 4 años de escolaridad y las que son detentoras de un certificado de $4^{\text {a }}$ clase se llega a un $68.5 \%$, al que se siguen el $18,7 \%$ con el $6^{\circ}$ y el $9^{\circ}$ años de escolaridad y el $9.7 \%$ con el $12^{\circ}$ grado o formación profesional, quedando una tasa muy baja de mujeres con educación superior $(3.1 \%)$.

En resumen, las madres de los encuestados/as son menos escolarizadas que sus padreshombres. Esta constatación nos lleva a concluir que la femenización de la enseñanza superior es un fenómeno reciente, por lo que las mujeres mayores han sido, hasta el comienzo del siglo, afectadas con mayor intensidad por niveles educativos limitados. Interesa ahora saber el grado de 
movilidad escolar de los encuestados/as en relación a sus padres y madres, lo que nos es dado por los gráficos 6 y 7 :

\section{Grafico 6. Escolarización hombres encuestados vs la paterna y la materna (\%)}

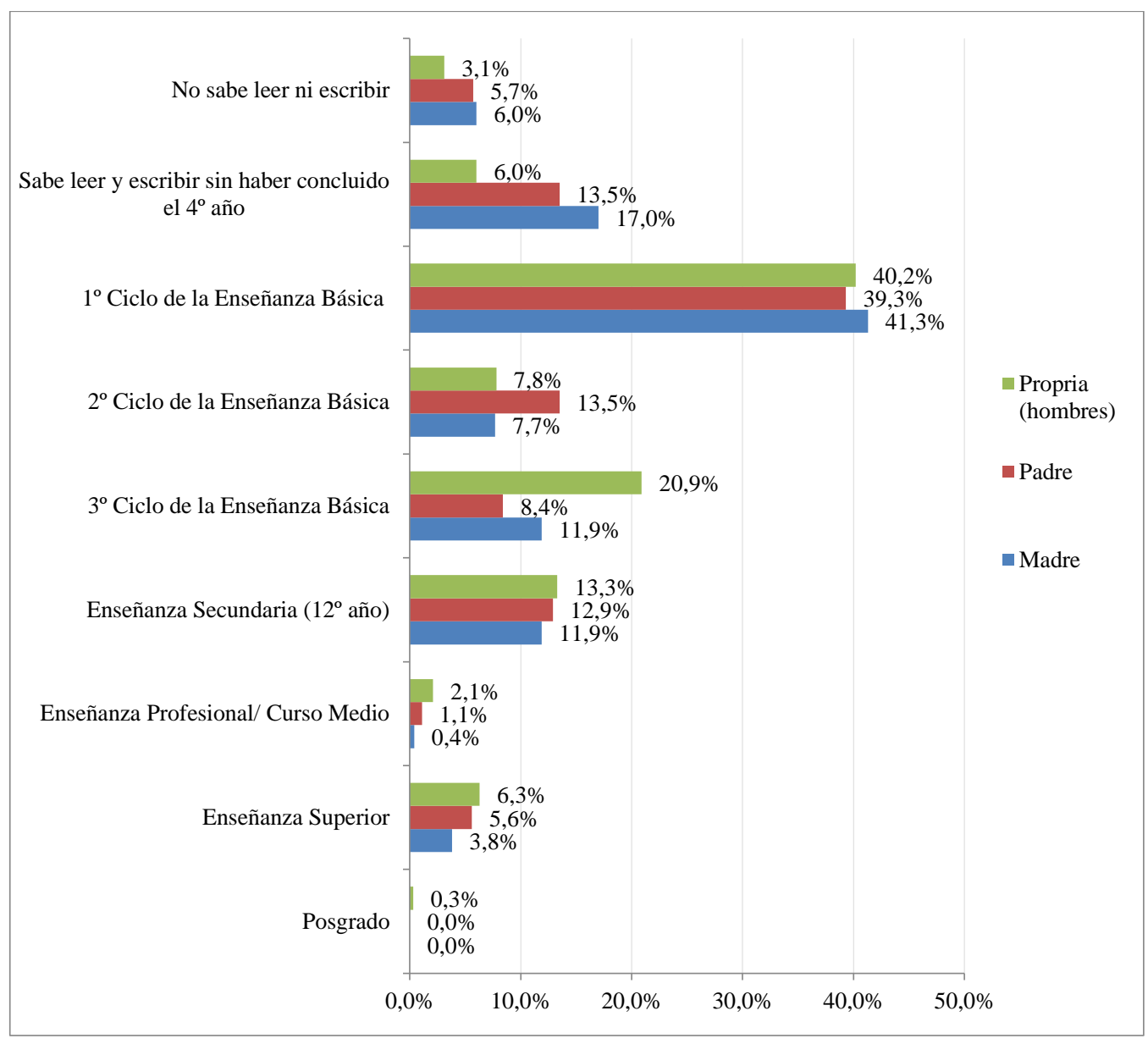

Fuente: IDG, 2011; N=371

Del gráfico 6 sobresale que, de forma general, los hombres encuestados tienen niveles de educación superiores a los del padre y particularmente a los de la madre, por lo que se observa mayor representatividad de los progenitores en las categorías más desprovistas de recursos educativos. Se notan igualmente tendencias similares de escolarización entre el padre y la madre de los encuestados, no evidenciándose disparidades acentuadas a este nivel. Sólo en la categoría que se refiere al segundo ciclo de la educación básica se percibe una diferencia alrededor del $6 \%$. El análisis relativo a las mujeres encuestadas muestra una situación ligeramente diferente, como lo evidencia el gráfico 7 : 


\section{Gráfico 7. Escolarización propia vs materna y paterna: mujeres (\%)}

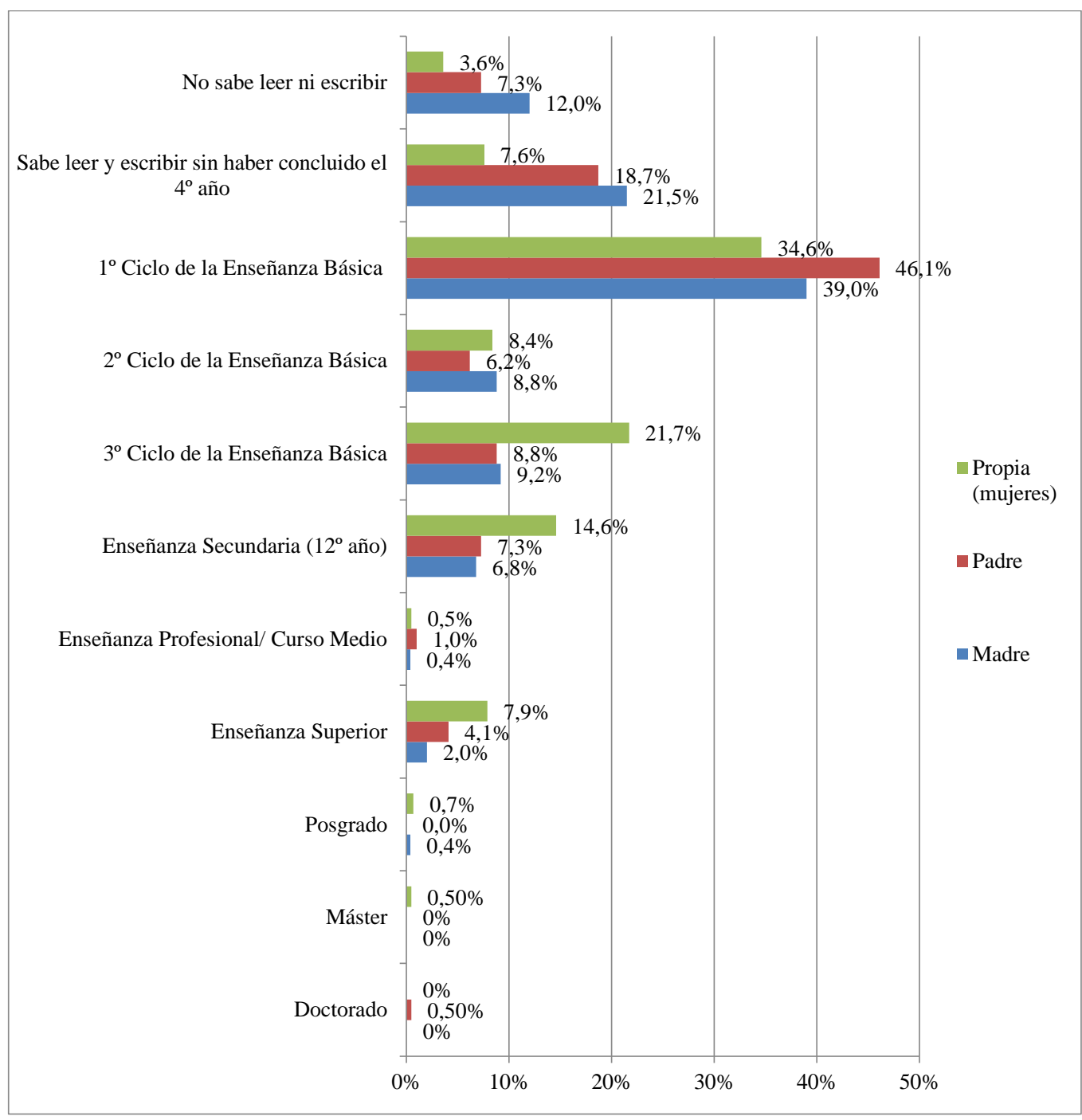

Fuente: IDG, 2011; N=371

En cuanto a la educación de los padres de los encuestados, se destaca, por un lado, tal como en el caso de los padres de los hombres, una concentración en los niveles inferiores de educación y, por otro, una menor representación en los niveles educativos más elevados en relación al gráfico 6. Se evidencia, como categoría modal, tanto para padres como para los propios encuestados, el tercer ciclo de enseñanza básica.

Cuestionadas las personas encuestadas relativamente a la escolarización de (ex)cónyuge, fue posible verificar que la mayoría posee escolarizaciones bajas o intermedias: además de el 1.9\% de analfabetos y el $9.2 \%$ que saben leer y escribir, pero no han completado el cuarto grado, hay el 
$28.11 \%$ con el cuarto grado, el $12,7 \%$ con el segundo ciclo y el $19.8 \%$ con el tercer ciclo, que suma un total de $71,7 \%$ con bajas cualificaciones. Ya todavía en el polo opuesto de escolarización media o elevada, hay que notar el 18,6\% con educación secundaria o escuela de curso medio/profesional, el $8.5 \%$ de graduados y el $1.2 \%$ con posgrado, incluyendo maestrías y doctorados (IDGPC, 2011). Si diferenciarmos por sexo, el gráfico 8 nos muestra los resultados obtenidos:

\section{Gráfico 8. Escolarización del (ex)cónyuge por sexo de los encuestados (\%)}

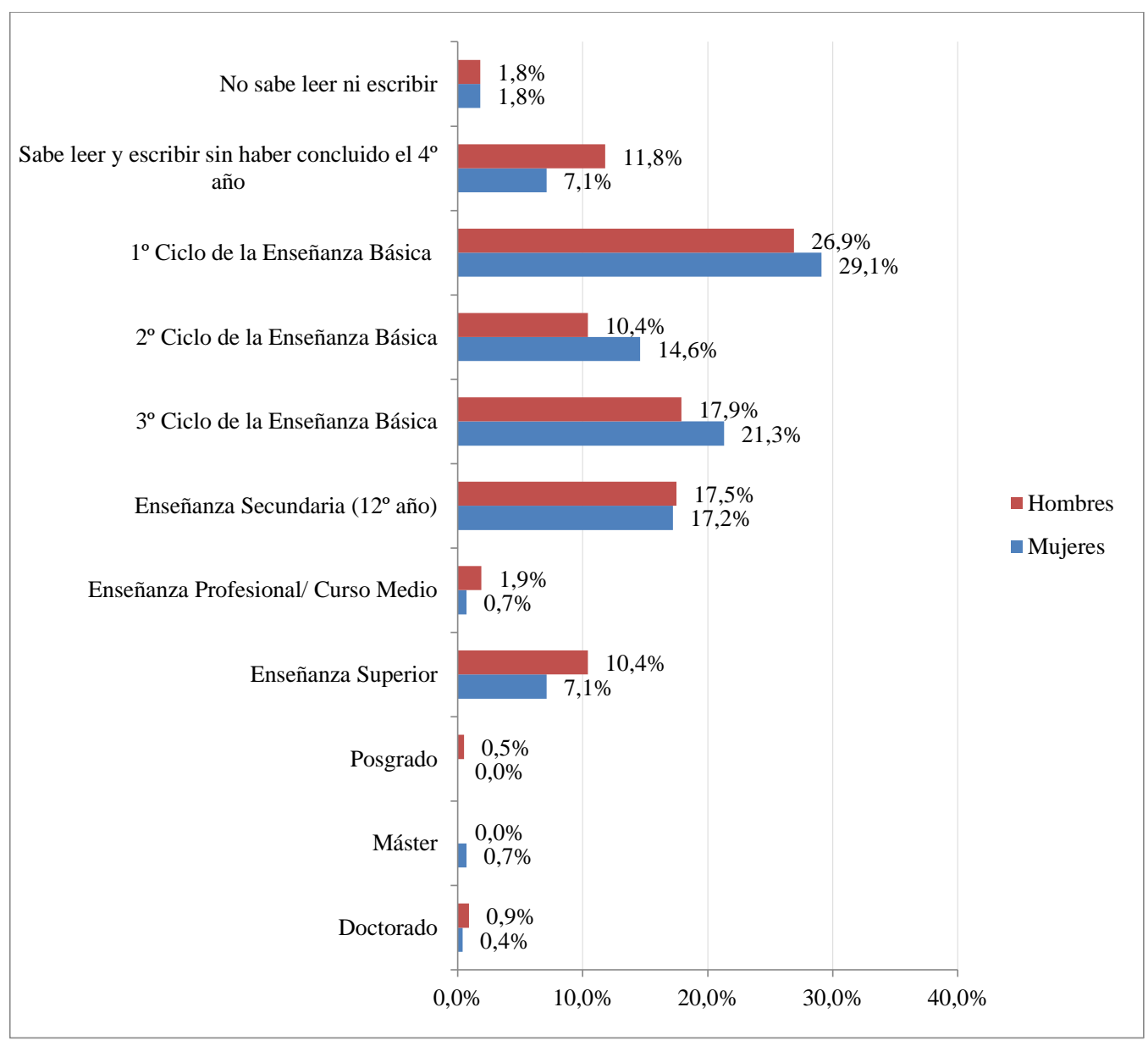

Fuente: IDG, 2011; N=480 (212 Homens; 268 Mulheres)

Los datos del gráfico 8, distribuidos por género, muestran que no hay diferencias considerables, anulándose uno al otro en la fila siguiente: por ejemplo, entre los que saben leer sin haber completado el cuarto grado ( $11,8 \%$ de los hombres vs $7.1 \%$ de las mujeres), la ventaja de los hombres de esta categoría es compensada en el primero, el segundo y tercer ciclos en estas mujeres por los hombres. Sin embargo, los hombres tienen un porcentaje más alto con respecto a 
la conclusión de la licenciatura, maestría y doctorado (11,8\% para los hombres vs $8.2 \%$ para las mujeres), no verificándose aún el efecto del mayor porcentaje de mujeres en grado y posgrado, en particular desde 1995.

Resultados similares se obtienen en los niveles de escolarización por género de los encuestados. Solicitados a declarar el nivel de escolarización, se verifica un mayor porcentaje de hombres en los extremos: ya sea en los dos últimos grupos de edad con menos de 4 años de escolarización ya sea los dos últimos niveles, o sea, el 12.0 año/enseñanza professional y en la educación superior. Siendo principalmente este último denotativo de la real desventaja de las mujeres, los resultados no pueden ser extrapolados para el número total de los encuestados y, mucho menos, en relación al universo de las mujeres en el país.

En el ámbito de esta investigación, se consideró apropiado observar la escolarización de los hijos de los encuestados.

Tabla 1. Escolarización de los hijos de los encuestados (\%)

\begin{tabular}{|l|c|c|c|c|c|c|}
\hline \multirow{2}{*}{ Escolarización } & \multicolumn{5}{|c|}{ Porcentaje de casos válidos } \\
\hline & Hijo 1 & Hijo 2 & Hijo 3 & Hijo 4 & Hijo 5 & Hijo 6 \\
\hline No sabe leer ni escribir & 12,2 & 10,9 & 8,5 & 11,4 & 8,3 & - \\
\hline $\begin{array}{l}\text { Sabe leer y escribir, pero no ha } \\
\text { concluído el 4 grado }\end{array}$ & 8,1 & 8,2 & 4,7 & 2,9 & 8,3 & - \\
\hline $1^{\circ}$ Ciclo de enseñanza básica & 10,0 & 10,6 & 17,9 & 17,1 & 33,3 & 33,3 \\
\hline $2^{\circ}$ Ciclo de la enseñanza básica & 11,6 & 14,0 & 17,9 & 28,6 & 33,3 & 33,3 \\
\hline $3^{\circ}$ Ciclo do enseñanza básica & 19,8 & 25,3 & 16,0 & 14,3 & - & 16,7 \\
\hline Enseñanza secundaria & 23,6 & 17,4 & 18,9 & 17,1 & 8,3 & - \\
\hline $\begin{array}{l}\text { Enseñanza profesional/curso } \\
\text { médio }\end{array}$ & 1,8 & 2,4 &, 9 & - & - & - \\
\hline Curso Superior (Licenciatura) & 11,8 & 10,2 & 13,2 & 8,6 & 8,3 & 16,7 \\
\hline PósGrado &, 4 & - & - & - & - & - \\
\hline Master &, 4 &, 3 & - & - & - & - \\
\hline Doctorado &, 2 &, 7 & 1,9 & - & - & - \\
\hline Total & 100,0 & 100,0 & 100,0 & 100,0 & 100,0 & 100,0 \\
\hline
\end{tabular}

Fuente: IDG; $1^{\circ}$ hijo: $N=497 ; 2^{\circ}$ hijo: $N=299 ; 3^{\circ}$ hijo: $N=109 ; 4^{\circ}$ hijo: $N=36 ; 5^{\circ}$ hijo: $N=13 ; 6^{\circ}$ hijo: $N=7$ 
Estos datos tendrán que ser vistos en contexto, porque hay resultados que pueden engañar como, por ejemplo, la alta tasa de niños que no pueden leer o escribir simplemente porque tienen menos de 7 años, pudiendo decirse lo mismo de otras filas de niños que aún no han completado su pasantía. En todo caso, los resultados relativos a niveles de educación básica y secundaria no difieren de los promedios nacionales.

Otra variante a tener en cuenta es la escolarización por grupos o bloques de años, lo que se expresa en la tabla 2:

Tabla 2. Escolarización de los hijos de los encuestados por años de escolaridad

\begin{tabular}{|l|c|c|c|c|r|c|}
\hline Escolaridad & \multicolumn{7}{|c|}{ Porcentaje de casos válidos } \\
\hline & Hijo 1 & Hijo 2 & Hijo 3 & Hijo 4 & Hijo 5 & Hijo 6 \\
\hline $\begin{array}{l}\text { Menos de 4 años de } \\
\text { escolarización }\end{array}$ & 20,4 & 19,1 & 13,2 & 14,3 & 16,7 & - \\
\hline $\begin{array}{l}4 \text { años de } \\
\text { escolarización }\end{array}$ & 10,0 & 10,6 & 17,9 & 17,1 & 33,3 & 33,3 \\
\hline $\begin{array}{l}\text { Entre 5 y 9 años de } \\
\text { escolarización }\end{array}$ & 31,4 & 39,2 & 34,0 & 42,9 & 33,3 & 50,0 \\
\hline $\begin{array}{l}12^{\circ} \text { ano/ Enseñanza } \\
\text { profesional }\end{array}$ & 25,5 & 19,8 & 19,8 & 17,1 & 8,3 & - \\
\hline Enseñanza superior & 12,8 & 11,3 & 15,1 & 8,6 & 8,3 & 16,7 \\
\hline Total & 100,0 & 100,0 & 100,0 & 100,0 & 100,0 & 100,0 \\
\hline
\end{tabular}

Fuente: IDG; $1^{\circ}$ hijo: $N=497 ; 2^{\circ}$ hijo: $N=299 ; 3^{\circ}$ hijo: $N=109 ; 4^{\circ}$ hijo: $N=36 ; 5^{\circ}$ hijo: $N=13 ; 6^{\circ}$ hijo: $N=7$

El alcance de la tabla 2 es igualmente limitado en la medida en que bastantes de los porcentajes en los primeros niveles incluyen no sólo casos reales de baja escolarización, sino también casos reales de niños y jóvenes que aún no han empezado todavía la escolarización o están en proceso de escolarización.

Si queremos ilustrar los niveles de educación en cuanto al primer hijo, se constata, en efecto, que los primeros hijos con 5 y 9 años de escolarización $(31,4 \%)$ y el $12^{\circ}$ grado o educación profesional $(25,5 \%)$ totalizaron $56,9 \%$, siendo todavía significativas las tasas de primeros hijos de parejas o uniones de hecho con 4 años de escolarización (10\%) o menos (20,4\%), sumando un total de $30.4 \%$. Además, es baja aún la tasa de personas con educación superior: con $12.8 \%$.

A pesar de sobresalir una progresiva inclusión de las mujeres en el sistema educativo, este logro no encuentra continuidad en la transición para el mercado laboral en el ámbito del capitalismo, tal como lo han sostenido teórica e empiricamente Hartman (1982), Ferreira (1993), Wall y Amâncio (2007) y Schouten (2011). Es aún persistente la segregación profesional con 
base en el género especialmente en el tipo de posiciones, funciones y remuneración recibida, precaridad laboral o incluso desempleo: "el hecho de ser hombre o mujer hace que el mismo diploma de enseñanza superior asuma valores diferenciados de utilización en la vida laboral" (Alves, 2004: 243). Subsiste, por lo tanto, una asociación entre género y transición hacia el trabajo. De hecho, las mujeres, aunque cada vez más cualificadas, están más expuestas al desempleo, a niveles de remuneración inferiores y a vínculos contractuales marcados por la incertidumbre de la continuidad del trabajo, por lo que, en la práctica, el valor del diploma en la enseñanza superior está fuertemente condicionado por el género de su portador, como observa $\mathrm{N}$. Alves (2005: 120):

"A pesar de este crecimiento continuo del número de graduadas de educación superior, es importante destacar que el ejemplo más obvio de que el diploma de educación superior no tiene un valor universal radica en las diferencias que se verifican entre graduados y graduadas".

La inserción de mujeres en el mercado laboral está lejos de ser una integración plena o incluso igualitaria por comparación a la de los pares masculinos. En este marco, la variable género es así co-explicativa de muchos de los procesos que se desarrollan (y perpetúan) dentro de las dinámicas laborales. Aún así, hay que tener siempre en cuenta variables tales como el nivel educativo, la clase social y las expectativas y valores, de forma que se comprendan más profundamente las desigualdades de género. Por otra parte, las jóvenes diplomadas son más afectadas por trabajos precarios y por el riesgo del paro, especialmente en tiempos de crisis, tal como lo refiere Gonçalves (2005:133):

“[...] están más fragilizadas en el mercado de trabajo en la medida en que ocupan predominantemente empleos poco cualificados y contractualmente precarios, los cuales son objeto privilegiado de reducción en tiempos de crisis económica y de contracción de los costes salariales de las organizaciones".

\section{Conclusión}

Con base en los resultados de la investigación y procurando sintetizar las conclusiones más relevantes de este texto fue posible ofrecer un panorama sobre la natureza sócio-jurídica de los hogares, donde sobresale un elevado porcentaje de personas con casa propia; se recogieron las representaciones de (auto)clasificación socio-económica, por parte de los encuestados/as, sobre la condición o estatuto de la familia de origen, así como de los propios encuestados, sea por nivel de matrimonio, sea en la actualidad, evidenciando algunas mejorías globales. 
Así mismo, ha sido posible, además de constatar el atraso relativo en la educación en comparación con otros países, trazar un mapa de itinerarios escolares a nivel intergeneracional, en el que se observa una gradual movilidad educativa ascendente de los encuestados en relación con sus padres. Más aún, no obstante se verificaren, a lo largo del tiempo y hasta las últimas décadas, asimetrías de género en detrimento de las mujeres, se comprueba, de hecho, en la actualidad una movilidad educativa ascendente más intensa entre las mujeres, expresada en una tasa de cualificaciones superiores más elevada que entre los jóvenes-hombres, incluso cuando, como lo evidencian varios estudios, tal no se traduce en posiciones de liderazgo y poder en la economía, en la sociedad y en las instituciones.

\section{BIBLIOGRAFÍA}

- Althusser, Louis [1972] (1965): Pour Marx, Paris: Maspero.

- Aves, Mariana Gaio (2004): “Os diplomados do Ensino Superior: diferenciação sexual nos processos de inserção profissional”. En: Sociologia - Revista da Faculdade de Letras, vol. 14, pp. 227-250.

- Alves, Natália (2005): Trajectórias Académicas e de Inserção Profissional dos Licenciados 1999-2003. Lisboa: Reitoria da Universidade de Lisboa.

- Araújo, Helena Costa (2000): Pioneiras na Educação - as Professoras Primárias, Contextos, Percursos e Experiênicas em Portugal, 1870-1933. Lisboa: Instituto de Inovação Educacional.

- Araújo, Helena Costa (2010): "Escola e construção da igualdade no trabalho e no emprego". En: Virgínia Ferreira (org): A igualdade de mulheres e homens no trabalho e no emprego em Portugal. Políticas e Circunstâncias. Lisboa: Comissão para a Igualdade no Trabalho e no Emprego, pp. 217-245.

- Bader, Veit y Benschop, Albert (1988): Ongelijkheden, Groningen: Wolters Noordhoff.

- Bell, Daniel (1977): O advento da sociedade pós-industrial. Uma tentativa de previsão social. São Paulo: Editora Cultrix.

- Benschop, Albert (1993): Klassen. Ontwerp van een transformationele klassenanalyse. Groningen: Wolters-Noordhoff.

- Bertaux Daniel (1977): Destinos sociais e estrutura de classe. Lisboa: Moraes Editora.

- Bordieu, Pierre (1980): Le sens pratique. Paris: Minuit.

- Bordieu, Pierre y Passeron, Jean Claude [1970] (1975): A reprodução: elementos para uma teoria do sistema de ensino. Rio de Janeiro: Francisco Alves.

- Burawoy, Michel (1979): Manufacturing Consent, Chicago e Londres. University of Chicago Press. 
- Chagas, Margarida y Perista, Margarida (2010): "Trinta anos de educação, formação e trabalho: convergências e divergências nas trajectórias de mulheres e homens". En: Virgínia Ferreira (org): A igualdade de mulheres e homens no trabalho e no emprego em Portugal. Políticas e Circunstâncias. Lisboa: Comissão para a Igualdade no Trabalho e no Emprego, pp. 191-216.

- Dahrendorf, Ralf (1959): Class and Class Conflict in Industrial Society. Londres: Routledge \& Kegan Paul.

- Dahrendorf, Ralf (1990): “A New Social Contract”. En: Ralf Dahrendorf (org): The Modern Social Conflict - An Essay in the Politics of Liberty. Berkeley y Los Angeles: University of California Press, pp. 166-194.

- Davis, Kingley y Moore, Wilbert E. [1945] (1976): “Alguns princípios de estratificação". En: Otávio Guilherme Velho et al. (orgs.): Estrutura de classes e estratificação social. Rio de Janeiro: Zahar Editores, pp. 115-132.

- Estanque (1999): Entre a fábrica e a comunidade. Porto: Afrontamento.

- Estanque, Elísio y Mendes, José Manuel (1997): Classes e desigualdades sociais em Portugal. Um estudo comparative. Porto: Afrontamento.

- Ferreira, Virgínia (1993): "Padrões de segregação das mulheres no emprego - uma análise do caso português no quadro europeu”. En: Boaventura de Sousa Santos (org): Portugal: um retrato singular. Porto: Afrontamento, pp. 231-257.

- Giddens, Anthony (1981): A estrutura de classes nas sociedades avançadas. Rio de Janeiro: Zahar Editores.

- Goffman, Erving (1974): Les rites d'interaction. Paris, Éditions du Minuit.

- Goldthorpe, John (1987): Social Mobility and Class Structure in Modern Britain. Oxford: Oxford University Press.

- Gonçalves, Carlos Manuel (2005): "Evoluções recentes do desemprego em Portugal”. En: Sociologia, Revista da Faculdade de Letras da Universidade do Porto, I série, vol. 15, pp. 125 164.

- Harris, Olivia e Young, Kate (1981): "Engendered Structures: Some Problems in the Analisys of Reproduction”. En: Joel S. Khan y Josep R. Llobera (orgs): The Anthropology of Pre-Capitalist Societies. Londres: MacMillan, pp. 109-147.

- Hartman, Heidi (1982): “Capitalism, Patriarchy and Job Segregation by Sex”. En: Anthony Giddens y David Held (orgs): Classes, Power and Conflict. Londres: MacMillan Education Ltd., pp. 446-469.

- Marques, Ana Paula; Silva, Manuel Carlos y Veiga, Carlos (2006): Assimetrias de Género e Classe. O caso das empresas de Barcelos. Barcelos: Kerigma.

- Marx, Karl [1867]: (1974): O Capital. Lisboa: Delfos. 
- Merton, Robert (1970): Sociologia: teoria e estrutura. São Paulo: Editora Mestre Jou.

- Parkin, Frank (1979): Marxisme and Class Theory: a Bourgeois Critique. Londres: Tavistock Publications.

- Parsons, Talcott [1951] (1988): El sistema social. Madrid: Alianza Editorial.

- Poulantzas, Nicos (1975): Classes in Contemporary Capitalism. Londres: NLB.

- Resende, José Manuel y Vieira, Maria Manuel (2003): Educação. Portugal Social 19902001:69-90. Lisboa: INE.

- Schouten, Maria Joana (2011): Uma Sociologia do Género. Vila Nova de Famalicão: Edições Húmus.

- Sebastiâo, João (1998): "Portugal, que modernidade? Os dilemas da escolaridade, Universalização, diversidade e inovação”. En: José Manuel Leite Viegas y António Firminio da Costa (orgs): Portugal, que modernidade?. Oeiras: Celta, pp. 311-332.

- Silva, Manuel Carlos (1998): Resistir e Adaptar-se. Constrangimentos e estratégias camponesas no Noroeste de Portugal. Porto: Afrontamento. . (2009): Classes Sociais: condição objectiva, identidade e acção colectiva. Vila Nova de Famalicão: Húmus.

- Wall, Karin y Amancio, Lígia (2007): Família e Género em Portugal. Lisboa: Imprensa de Ciencias Sociais.

- Weber, Max [1920] (1978): Economy and Society. Editado por Guenther Roth y Claus Wittich. Berkeley y Londres: University of California Press.

- Wright, Eric Olin (1985): Classes, Londres. Nova Iorque: Verso. 